\title{
Web Application Performance Analysis of E- Commerce Sites in Bangladesh: An Empirical Study
}

\author{
Mahfida Amjad ${ }^{1}$, Md. Tutul Hossain ${ }^{2}$, Rakib Hassan ${ }^{3}$, Md. Abdur Rahman ${ }^{4}$ \\ ${ }^{1,2,3}$ Department of Computer Science \& Engineering, Stamford University Bangladesh, Bangladesh \\ ${ }^{4}$ Centre for Advanced Research in Sciences, University of Dhaka, Dhaka, Bangladesh \\ Email: ${ }^{1}$ mahfidaamjad@ stamforduniversity.edu.bd, ${ }^{2}$ tutulhossain.cse@gmail.com, \\ ${ }^{3}$ tipusultan9t7a@gmail.com, ${ }^{4}$ mukul.arahman@gmail.com
}

Received: 23 November 2020; Accepted: 20 January 2021; Published: 08 April 2021

\begin{abstract}
The users of e-commerce sites are growing rapidly day by day for easy internet access where the performance of web applications plays a key role to satisfy the end-users. The performance of these websites or web applications depends on several parameters such as load time, fully loaded (time), fully loaded (requests), etc. This research tries to investigate and find out the parameters that affects the web performance and it has been tested on ecommerce applications of Bangladesh, where eleven parameters are considered and these are fully loaded (requests), first CPU idle, speed index, start render, load time, fully loaded (time), document complete (time), last painted hero, first contentful paint, and first byte. According to the analysis some applications need to take care of or the developers need to re-modify it. As per the investigation of scanned information, the applications fall under three classes. To start with, the applications do not demonstrate acceptable records to be investigated. The second and third classification applications required medium and high reaction times at the user end separately. Also, the fully loaded (requests)' and document complete (requests) show the most noteworthy required time at the user end, where maximum values are 347 and 344 seconds individually.
\end{abstract}

Index Terms: Quality of Experience Web, DOM, onLoad, TTFB, TTFP, Speed Index, Byte Index, Performance Testing, Testing Tools.

\section{Introduction}

Web services are widely utilized in most applications. The amount of users is increasing rapidly and applications enable the users with simultaneous access to the system. Therefore, the requirement for performance testing is additionally becoming important. Performance testing could be a non-functional variety of testing that has the stakeholders with analysis supported speed, stability, and scalability of the application.

Performance testing is employed to spot bottlenecks and ensure good software quality to the users. Common problems faced while conducting performance testing are test environment, testing tool selection, ambiguous requirements, unavailability of valid data, etc. There is a good sort of performance testing tools available within the market. The choice of tools for testing will depend upon factors like license cost, hardware requirements, platform support, etc. Generic performance testing process includes the subsequent steps:

- Identifying testing environment.

- Identifying performance acceptance criteria.

- Planning \& designing performance tests

- Configuring the test environment

- Implementation of test design.

- Run the tests

- Analyze, tune and retest [1].

Performance testing is important before the arrival of any sort of application. It guarantees improved consumer loyalty, steadfastness, and maintenance. The presentation of an online application is estimated by a few accessible testing apparatuses. 
This paper will attempt to discover the accompanying inquiries

- What are the key highlights or boundaries of execution examination of any internet business webpage?

- Which boundaries influence most the client experience of sites or web applications?

- How to distinguish the reaction season of the framework under the expanding heaps of the clients?

Furthermore, subsequently, the destinations of this examination can be plot as follows-

- To distinguish the boundaries for execution assessment of online business locales.

- To recognize the most influenced boundary for the client experience of online business locales.

- To recognize the reaction season of the web applications for assessing execution.

In this paper, fully and partially open source tools have been used to scan some e-commerce applications. Web applications providing e-commerce services for an outsized number of shops in Bangladesh are considered to be analyzed. The chosen ten applications include small, medium, and huge level e-commerce service, providers. And this paper is not mentioning the names of the e-commerce sites considering the privacy issues. The performance of the websites is measured supported eleven parameters and these are fully loaded (requests), first CPU idle, speed index, start render, load time, fully loaded (time), document complete (time), last painted hero, first contentful paint, first byte. The numeric data has been collected using three reputed webpage performance testing tools WebPageTest, PageSpeed Insights and Gtmetrix.

According to the analysis this paper finds that fully loaded (requests) and document complete (requests) parameters indicate highest required time at users-end, where maximum values are 347 and 344 seconds respectively.

The rest of this paper is organized as follows; Section 2 discusses the related work. Section 3 is about the parameters of performance estimation of web applications. Section 4 and 5 illustrates the empirical data collection methodology, findings and data analysis respectively. Finally, Section 6 concludes this paper with conclusion.

\section{Related Work}

The knowledge and application tools to automate performance testing are important for ensuring software reliability and quality. This section will discuss some existing works of performance analysis of websites.

A user behavior model has been proposed based on the log file from user navigation in [3]. They used five indexes to measure the performance; these are service response time, service path length, service utilization, service implementation rate and access error rate. A web performance simulation with trend analysis of the system under test (SUT) has been done in [2]. For achieving this system requires input information, like Monitoring Points and StaticInformation about the SUT. The paper [5] investigated some performance testing tools i.e. Apache JMeter, NeoLoad, and Loadster is done based on usability test parameters \& performance parameters. System performance testing indicators, performance testing methods and performance optimization strategies are discussed in [4] based on three aspects: browser access, server and data storage. An empirical analysis of e-commerce based web applications to evaluate the current web application security scenario in Bangladesh has been depicted in [6]. A static-analysis approach has been proposed in [10] that automatically constructs a model of each webpage during a given application. A page model may be a code fragment in the same language because the server-side code, which faithfully over approximates the possible elements of the page further because the control flows and data flows because of these elements. The server-side code in conjunction with the page models then becomes a typical (non-web) the program, thus amenable to analysis using standard code-based tools. In [11] it has shown that in a very controlled environment it can significantly enhance the performance of web applications employing a small set of performance enhancement techniques with a complete implementation time starting from 10 to 50 working hours for applications running on one multiple role (e.g. web, proxy, application) server. A resource allocation approach for performance testing for web applications has been introduced in [12] which maximize resource utilization and improve load balance between resources. It monitors tasks or resources status as fault handling strategies that can handle any failure occur whether a resource failure or failure during task execution. In [13] it examines quality of a website by using Webqual 4.0 methodology to see the importance of that quality using IPA (Importance Performance Analysis). To test microservice web application [14] uses the RESTful API and the RabbitMQ method under various numbers of users to compare and evaluate their performance in different circumstances. In [15] usability evaluation using four different evaluation methods Feedback Capture after Task (FCAT), Retrospective Think Aloud (RTA), Retrospective Think Aloud with Eye Movement (RTE) and observation has been performed for an ecommerce website with a group of potential consumers with age range of $18-22$. 


\section{Parameters for Performance Estimation of Web Applications}

Most Website performance testing tool measures the performance of some common parameters supported variety of tests. Web Performance Testing is executed to provide accurate information on the readiness of an application through testing the onsite and monitoring the server-side application.

\section{A. Load Time}

The Load Time is measured due to the time from the start of the initial navigation until the beginning of the window load event (on load). Page load time may be an internet performance metric that directly impacts user engagement and a business's bottom line. It indicates how long it takes for a page to totally load within the browser after a user clicks a link or makes a missive of an invite [7].

\section{B. First Byte}

The First Byte Time (TTFB) also known as First Byte measures the time elapsed between the moment an internet user makes an HTTP request, like loading a webpage, to the time the first byte is received by the client's browser [7].

\section{Start Render}

The Start Render time is measured because the time from the start of the initial navigation until the first non-white content is painted to the browser display. The start Render gives the time necessary for a primary element to render on the page, no matter its size or its importance for the user. This could be a background color, a text, or else, but in every case; a sturdy sign is distributed to the visitor: the page is rendering, operations are ongoing and also the rest of the content should come [7].

\section{Speed Index}

The Speed Index is expressed in milliseconds and obsessed with size of the view port. It is the common time at which visible parts of the page are displayed [9].

\section{E. Last Painted Hero}

Last Painted Hero might be an artificial metric that shows the user when the last piece of critical content is painted. It keeps reading to seek out how Last Painted Hero works, why (and how) the contents are created and therefore the way it can facilitate user's perceive relating the speed of the online pages [8].

\section{F. First CPU Idle}

First CPU Idle measures when a page is minimally interactive, or when the window is quiet enough to handle user input. Generally, it occurs when most, but not necessarily all visible UI elements are interactive, and also the program responds, on average, to most user input within 50ms [8].

\section{G. Document complete}

Document complete is also some extent where the browser on load event fires and is typically when all of the static page content has loaded [8].

\section{H. Fully Loaded Time}

The fully loaded time is measured due to the days from the beginning of the initial navigation until there are 2 seconds of no network activity after the document complete. This might usually include any activity that is triggered by JavaScript after the foremost page loads [8].

\section{Requests}

This is the quantity of requests that had to be made by the browser for pieces of content on the page (images, JavaScript, CSS, etc.)It is an information request message from a client to a server over the hypertext transfer protocol (HTTP). For images, text, or pages to be delivered to the user browser, it has to request to fetch that data and uses an HTTP request to try and do exactly that [2].

\section{J. Bytes In}

Bytes in is the amount of information that the browser had to download to load the page. It is also commonly mentioned as Page Size [2].

\section{K. Time to Interactive}

Time to Interactive (TTI) is a non-standardized web performance progress metric defined as the point in time when the last long task finished and was followed by 5 seconds of network and main thread inactivity. Time to interactive 
gives users a deeper insight into how the website is perceived on the web site user's side of the browser [2].

\section{Max Potential First Input Delay}

First input delay measures the time from when a user first interacts together with your site, like clicking a button, to the time when the browser is truly ready to answer that interaction [2].

\section{First Meaningful Paint}

First meaningful paint is that the time it takes for a page's primary content to look on the screen. It is used as an approximation of first meaningful paint but it often catches non-meaningful paints like headers and navigation bars [2].

\section{N. Largest Contentful Paint}

Largest contentful paint (LCP) is a crucial, user-centric metric for measuring perceived load speed as it marks the page load timeline when the page's main content has been loaded [2].

\section{O. Cumulative Layout Shift}

Cumulative layout shift measures the assemblage of all individual layout shift scores for each unexpected layout shift that happens during the complete lifespan of the page. A layout shift occurs any time a visual element changes its position from one frame to the subsequent [2].

\section{Empirical Data Collection}

The web application performance metrics play vital role for having good user experience. The good performance increases usability of the application as well as user satisfaction. To this end, performance related data are collected using different tools for e-commerce web applications in Bangladesh.

Several enterprise e-commerce web applications are scanned with different web tools named as webpage test, google page speed insights and gtmetrix. The data are analyzed based on different performance ability parameters such as load time, start render, speed index, largest contentful paint, etc.

\section{A. Performance Data Scanning Tool Selection}

Performance of a web application can be measured by several available testing tools. In this research, fully and partially open source tools are used to scan large e-commerce applications. Among the available web testing tools WebPage Test, Google Page Speed Insights, Gtmetrix are the prominent which are selected and used in this investigation. The short description regarding these tools is given below.

- Webpage Test: Webpage test is one of the most popular and free tool for measuring webpage performance. This tool supports web performance testing on users' site from different locations across the world using different browsers.

- PageSpeed Insights: The web page insight score is identified with PageSpeed Insights tool. This performance report is generated from a page on both mobile and desktop devices. Using the scanned report, suggestions can be provided on how that page may be improved.

- Gtmetrix: This is an excellent tool to detect page speed information using different location, browsers, connection speed and more. Those collected reports are analyzed to make web page faster than previous. This is a free tool for web page speed performance analysis using Google Page Speed and YSlow.

\section{B. E-Commerce Web Application Selection}

Web applications providing e-commerce services for large number of retailers in Bangladesh are considered to be analyzed. The selected applications includes small, medium and large level e-commerce service providers where varieties of technologies are used as development frameworks and tools such as Google Analytics, Google Tag Manager, Nginx, Web pack, Criteo, Ink, React, Tengine, Zepto, jQuery, Express, Node.js, Font Awesome, Google Font API, IIS, Microsoft ASP.NET, OWL Carousel, Sweet Alert, Twitter Bootstrap, jQuery UI, Windows Server, Cloud Flare, Crazy Egg, Ion icons, Algolia Real-time Search, PHP, Apache, CentOS, Magento, Prototype, script.aculo.us, NopCommerce, Light box, jQuery Mobile, Payment gateway.

\section{Environment Setup}

The workstation with $2.00 \mathrm{GHz}$ Intel core i7 CPU, and 6GB primary memory, Windows $10 \mathrm{OS}$ and $15 \mathrm{Mbps}$ internet connection are used for this empirical data collection. However, CPU $2.00 \mathrm{GHz}$ Intel core i5 or higher, RAM 6GB or higher, GPU not depended on GPU, HDD free space or higher and Internet Speed 10 Mbps or higher setup is recommended. 
Table 1. Performance Evaluation Based on 11 Parameters Related to Response Time (in seconds) of 10 Sites

\begin{tabular}{|c|c|c|c|c|c|c|c|c|c|c|}
\hline Performance & Site $_{1}$ & Site $_{2}$ & $\mathrm{Site}_{3}$ & Site $_{4}$ & Site $_{5}$ & Site $_{6}$ & Site $_{7}$ & Site $_{8}$ & Site $_{9}$ & Site $_{10}$ \\
\hline Load Time & 8.16 & 8.77 & 21.10 & 12.47 & 10.94 & 11.47 & 1.53 & 0.18 & 23.42 & 1.61 \\
\hline First Byte & 1.86 & 3.21 & 1.76 & 1.61 & 2.80 & 2.05 & 0.40 & - & 2.40 & - \\
\hline Start Render & 3.60 & 3.80 & 2.70 & 1.80 & 3.80 & 4.30 & 0.80 & - & 4.20 & - \\
\hline $\begin{array}{c}\text { First Contentful } \\
\text { Paint }\end{array}$ & 3.60 & 3.81 & 2.67 & 1.74 & 3.78 & 4.23 & 0.80 & - & 4.18 & - \\
\hline Speed Index & 6.46 & 6.41 & 4.58 & 6.46 & 6.37 & 7.43 & 0.94 & - & 10.43 & - \\
\hline Last Painted Hero & 11.80 & 10.90 & 6.50 & 13.00 & 11.00 & 20.50 & 1.20 & - & 24.50 & - \\
\hline First CPU Idle & 5.21 & 9.38 & 15.88 & 12.33 & 7.64 & 11.35 & 0.80 & - & 10.27 & - \\
\hline $\begin{array}{c}\text { Document Complete } \\
\text { (Time) }\end{array}$ & 8.16 & 8.77 & 21.10 & 12.47 & 10.94 & 11.47 & 1.53 & - & 23.42 & - \\
\hline $\begin{array}{l}\text { Document Complete } \\
\text { (Requests) }\end{array}$ & 82 & 87 & 122 & 313 & 123 & 264 & 31 & 0 & 344 & 0 \\
\hline Fully Loaded (Time) & 9.69 & 11.68 & 22.89 & 12.47 & 11.31 & 18.49 & 2.77 & 0.18 & 24.61 & 1.61 \\
\hline $\begin{array}{l}\text { Fully Loaded } \\
\text { (Requests) }\end{array}$ & 88 & 106 & 129 & 313 & 126 & 288 & 46 & 1 & 347 & 2 \\
\hline Average & 220.38 & 250.96 & 329.08 & 687.88 & 306.64 & 631.82 & 86.24 & 1.18 & 795.01 & 3.61 \\
\hline
\end{tabular}

\section{Findings and Data Analysis}

In this section, the collected performance related information has been analyzed in order to find the major problems in web pages. Use performance test results help stakeholders to make architectural and business decisions. The 10 top most e-commerce platforms have been scanned with mentioned 3 different tools. This paper is not disclosing the names of the e-commerce sites considering privacy issues.

The comparison analysis of performance data for e-commerce platform applications was plotted in Table 1 and Table 2. Eleven parameters related to response-time (in second) are shown in Table I and two parameters related to response with bytes-in are presented in Table 2 where, the heading $\mathrm{Site}_{2}, \mathrm{Site}_{1}$, Site ${ }_{3}$, etc. indicates application-1, application-2 application-3, etc and the performance parameters are given in the row. The value represents in second. The web applications performance comparison analysis also depicted in Fig. 1.

\section{A. Performance Parameters}

Eleven performance parameters such as fully loaded (requests), first CPU idle, speed index, start render, load time, fully loaded (time), document complete (time), last painted hero, first contentful paint, first byte has been considered to collect web page reports using discussed tools. It shows that eight applications out of ten satisfied all the parameters. Two applications record only load time, fully loaded (time) and fully loaded (requests) parameters.

According to Table 1, 23.42 second is highest value for load time parameter and 0.18 second is the lowest. Where, the highest and lowest value of First byte is 3.21 and 0.40 second respectively. The value range of start render parameter is 0.80 to 4.30 second. The speed index records 10.43 and 0.94 seconds as the highest and lowest score respectively. Last painted hero and first CPU idle show lowest value 1.20 and 0.80 seconds respectively where, highest scores are 24.50 and 15.88 seconds respectively.

Document complete (time) performance score falls between 1.53 and 23.42 seconds as lowest and highest time respectively. The document complete (requests) parameter indicates higher score 344 seconds compare to others except one, where lowest required second also reported for this parameter which is 0 seconds. However, it is indicating as outlier record, as document complete request must take few seconds.

Fully loaded (time) represents 0.18 and 24.61 seconds as lowest and highest value respectively. This parameter is covered by all the applications. However, only three applications among ten records less than 3 seconds as fully loaded time. At last, the fully loaded (requests) reports highest time compare to other parameters for investigated applications.

The Table 2 presents document complete and fully loaded performance records where 1 to 10 represents the sites ( 1 for site-1, 2 for site-2, etc.). As both fields represents data in kilo bytes, shows in separate table in order to make clear comparison between those. The document complete bytes are always higher than fully loaded record except for one site which indicates, the sites take more time to fully loaded on user end. 
The average score for each site shown in Table 1, where 1.18 shows as lowest value. However, that application did not covered by maximum parameters. The applications which records value for all parameters, the lowest time are 86.24 seconds. On the other hand, the highest average score is 795.01.

The numeric data in Table 1 and 2 was collected using WebPageTest, PageSpeed Insights and Gtmetrix tools. All 3 tools are reputed reliable for webpage performance testing. Even though, all tools does not produce results for all parameters. The results were measured with average value of each parameter. Also, for each parameter, the system runs 3 times and collected results. Then, the average of 3 results used a value for performance parameter to ensure accuracy. For example: Load time of Response- $1=8.17$, Response- $2=8.14$ and Response- $3=8.15$. And hence the Average is 8.16 .

Table 2. Performance records (in bytes) Based on Document Complete and Fully Loaded Parameters

\begin{tabular}{|c|c|c|}
\hline Sites & $\begin{array}{c}\text { Document Complete } \\
\text { (Bytes In) }\end{array}$ & $\begin{array}{c}\text { Fully Loaded } \\
\text { (Bytes In) }\end{array}$ \\
\hline 1 & $2,017 \mathrm{~KB}$ & $2,026 \mathrm{~KB}$ \\
\hline 2 & $1,554 \mathrm{~KB}$ & $1,799 \mathrm{~KB}$ \\
\hline 3 & $1,793 \mathrm{~KB}$ & $1,819 \mathrm{~KB}$ \\
\hline 4 & $3,599 \mathrm{~KB}$ & $3,599 \mathrm{~KB}$ \\
\hline 5 & $3,301 \mathrm{~KB}$ & $3,328 \mathrm{~KB}$ \\
\hline 6 & $5,330 \mathrm{~KB}$ & $7,819 \mathrm{~KB}$ \\
\hline 7 & $342 \mathrm{~KB}$ & $409 \mathrm{~KB}$ \\
\hline 8 & $0 \mathrm{~KB}$ & $3 \mathrm{~KB}$ \\
\hline 9 & $7,876 \mathrm{~KB}$ & $7,879 \mathrm{~KB}$ \\
\hline 10 & $0 \mathrm{~KB}$ & $2 \mathrm{~KB}$ \\
\hline
\end{tabular}

The web applications performance testing record has been depicted in Fig. 1, where apps and required time (in second) are shown vertically and horizontally respectively. The figure is representing ten sites with eleven performance parameters for each where, Site $_{8}$ and $\mathrm{Site}_{10}$ does not show satisfactory scan record for analysis. Among the others, Site ${ }_{1}$, Site2, and $\mathrm{Site}_{7}$ fall under medium range for required time (in second) at user end. However, Site 4 , Site 6 and $\mathrm{Site}_{9}$ fall under high range for required responding time (in second) at user browsers.

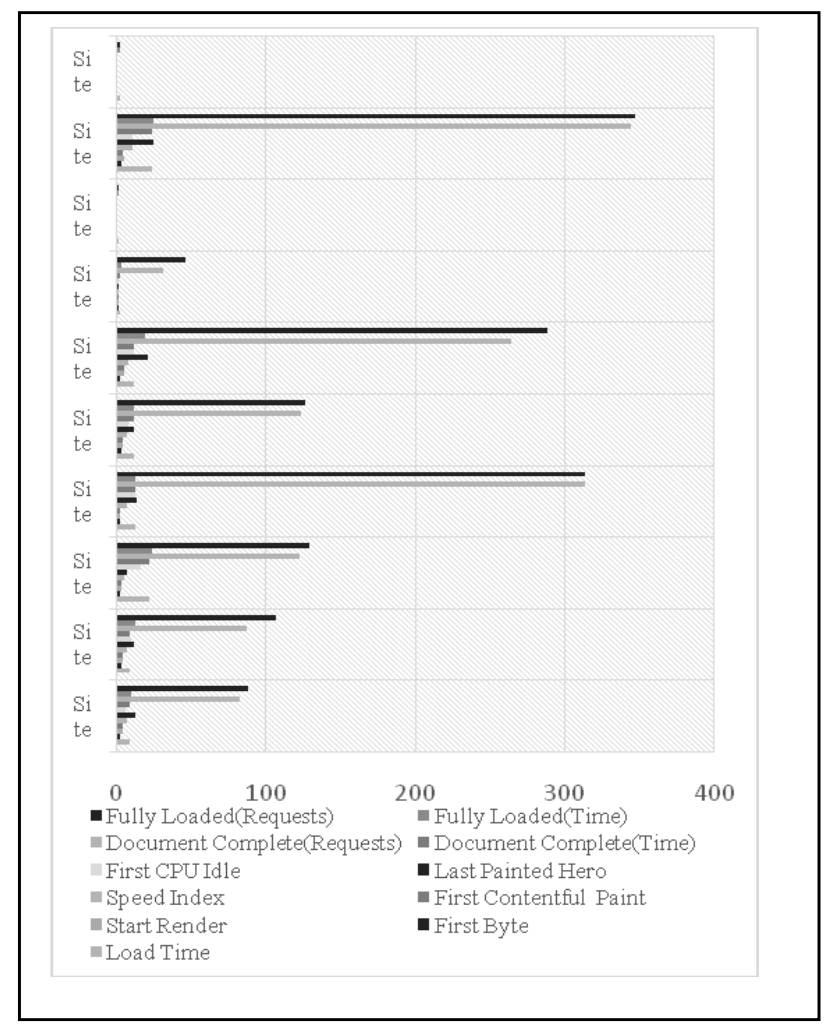

Fig. 1. Comparative analysis of the parameters 
According to Table 1, Table 2 and Fig. 1, it is clear that fully loaded (requests) and first CPU Idle response time is taking more for most of the tested applications where fully loaded (bytes-in) show more memory occupies compare to document complete (bytes-in). However, few applications development framework does not support for performance testing with available tools.

\section{Conclusion}

E-commerce platforms are growing rapidly for its popularity in the Bangladesh using web based applications. The performance of an e-commerce application depends on the eleven parameters such as fully loaded (requests), first CPU idle, speed index, start render, load time, fully loaded (time), document complete (time), last painted hero, first contentful paint, and first byte which are calculated using response time (in second) and bytes-in. The customer satisfaction depends on applications' performance such as loading time, fully loaded (requests) etc. at end-user's browsers. This research investigated top ten e-commerce applications in Bangladesh to find out the user experiences considering eleven different parameters. According to the analysis of scanned data, the applications fall under three categories. First, the applications do not show satisfactory records to be analyzed. The second and third category applications required medium and high response time at user end respectively. And the fully loaded (requests)' and document complete (requests) parameters indicate highest required time at users-end, where maximum values are 347 and 344 seconds respectively. This research will try to develop an automated tool for measuring the performance of different ecommerce sits so that the developers and also the company can find the aspects where they have to focus to improve the performance of their sites.

\section{References}

[1] Dipika Kelkar, Kavita Kandalgaonkar, Analysis and Comparison of Performance Testing Tools, International Journal of Advanced Research in Computer Engineering \& Technology (IJARCET), Volume 4 Issue 5, May 2015.

[2] Martin Pinzger. "Automated Web Performance Analysis” Department of Telecooperation Johannes Kepler University Linz Austria, 978-1-4244-2188-6/08, page: 513-516, 2008.

[3] Quanshu Zhou, Hairong Ye and Zuohua Ding. "Performance Analysis of Web Applications Based on User Navigation" 2012 International Conference on Applied Physics and Industrial Engineering. Page: 1319 - 1328, 2012.

[4] Yu Yao and Jie Xia. "Analysis and Research on the Performance Optimization of Web Application System in High Concurrency Environment", Information Technology, Networking, Electronic and Automation Control Conference, IEEE, page 321-326, 2016.

[5] Divya Saharan, Yogesh Kumar, Dr. Rahul Rishi, "Analytical Study and Implementation of Web Performance Testing Tools" International Conference on Recent Innovations in Electrical, Electronics \& Communication Engineering (ICRIEECE), page: 2370-2377, 2018.

[6] Md. Abdur Rahman, Mahfida Amjad, Byezid Ahmed, Md. Saeed Siddik. "Analyzing Web Application Vulnerabilities: An Empirical Study on E-Commerce Sector in Bangladesh”, International Conference on Computing Advancements, ICCA 2020, Page: $10-12,2020$.

[7] Web Page test documentation, https://sites.google.com/a/webpagetest.org/docs/usingwebpagetest/metrics?fbclid=IwAR1aGCPW3sV-NCyB72pVbCeWzt2jQQB8fANfdweXKNJzwW7i4rxn4k68xmY

[8] Google's Page Speed insights tool, https://www.semrush.com/blog/how-to-improve-your-google-pagespeed-insightsscore/?fbclid=IwAR3-JtpQFQ6HBZLEKHUw9nKBwSMUjvyrt1YwzfHmTPzNgTwdksfNchElldk.

[9] Lubos Kmetko, Do Intro Animations Affect Perceived Performance? https://www.xfive.co/blog/intro-animations-perceivedperformance/

[10] Snigdha Athaiya, "Inferring Page Models for Web Application Analysis", Association for Computing Machinery, ACM, page: 10-14, 2017.

[11] Igor Jugo, Dragutin Kermek, Ana Meštrović, "Analysis and Evaluation of Web Application Performance Enhancement Techniques", International Conference on Web Engineering, ICWE, July 2014.

[12] Amira Ali, Nagwa Badr, "Performance Testing as a Service for Web Applications", IEEE Seventh international Conference on intelligent Computing and information Systems (ICICIS' 15), 2015.

[13] Johanes, Fernandes Andry , Kevin Christianto, Fuji Rahayu Wilujeng, "Using Webqual 4.0 and Importance Performance Analysis to Evaluate E-Commerce Website", Journal of Information Systems Engineering and Business Intelligence, page: 2331, 2019.

[14] Xian Jun Hong, Hyun Sik Yang, Young Han Kim. "Performance Analysis of RESTful API and RabbitMQ for Microservice Web Application", International Conference on Information and Communication Technology Convergence (ICTC), Page: 257$259,2018$.

[15] K. N. Goh , Y.Y. Chen , F.W. Lai , S.C. DaudandA. Sivaji, S.T.Soo, "A Comparison of Usability Testing Methods for an Ecomerce Website: A Case Study On A Malaysia Online Gift Shop", 10th International Conference on Information Technology: New Generations, Page: 143-150, 2013. 


\section{Authors' Profiles}

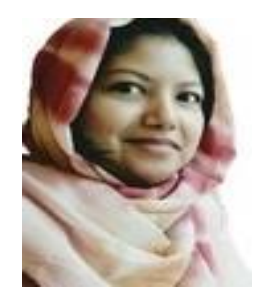

Mahfida Amjad was born in 1985 in Dhaka, Bangladesh. She has completed her Master degree in Information Technology from Institute of Information Technology from University of Dhaka in 2009. And she completed B.Sc. in Computer Science \& Engineering from Manarat International University in 2007. She is a faculty member of Computer Science and Engineering (CSE) Department of Stamford University Bangladesh. She has devoted herself in teaching profession since 2012. Her research area is wireless communication network, human computer interaction and software engineering. She has published a number of research papers in various international journals and conferences.

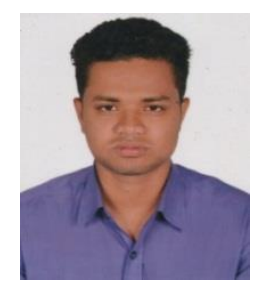

Md. Tutul Hossain was born in 1997 In Narail, Dhaka, Bangladesh. He has completed his B.Sc in ComputerScience and Engineering from Stamford University Bangladesh in 2020. Currently he is working on WordPress Developer at Buy Perfume in Bangladesh (BPIB).

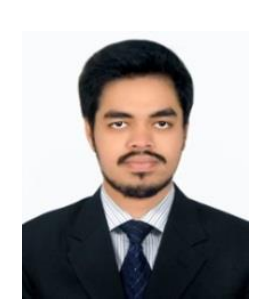

Rakib Hassan was born in 1997 in Dhaka, Bangladesh. He has completed his B.Sc degree in Stamford University.

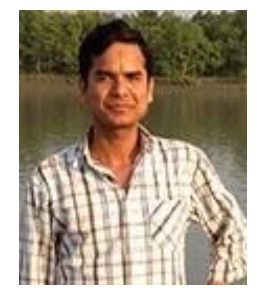

Md. Abdur Rahman received his BSc in Information Technology from Visva Bharati University, India in 2004 He has completed his Post Graduate Diploma and Master in Information Technology from University of Dhaka, Bangladesh, in 2008 and 2009 respectively. He is a Senior Computer Scientist in the Centre for Advanced Research in Sciences at the University of Dhaka. His major research interest includes text analytics, application of machine and deep learning in software engineering and natural language processing. He has published a number of research papers in various international journals and conferences.

How to cite this paper: Mahfida Amjad, Md. Tutul Hossain, Rakib Hassan, Md. Abdur Rahman, " Web Application Performance Analysis of E-Commerce Sites in Bangladesh: An Empirical Study", International Journal of Information Engineering and Electronic Business(IJIEEB), Vol.13, No.2, pp. 47-54, 2021. DOI: 10.5815/ijieeb.2021.02.04 